\title{
Chapter 3 \\ Unmanned Aerial Vehicles for Environmental Monitoring, Ecological Conservation, and Disaster Management
}

\author{
Raj Madhavan, Tatiana Silva, Flavia Farina, Rodrigo Wiebbelling, \\ Leonardo Renner and Edson Prestes
}

\subsection{Introduction}

Unmanned Aerial Vehicles (UAVs) or Drones have received unprecedented attention in the last few years. From Amazon's ambitious plan of its fleet of prime air package deliveries right to our doorsteps to United States Air Force Reapers firing hellfire missiles at targets in Afghanistan and Iraq to paparazzi's chasing celebrities to get that prized picture of a Hollywood star's baby, they have conjured up images only limited by our vivid imaginations. Whatever mode of control they are flown under (they can be either autonomous or remotely piloted by ground operators), for a destructive or a constructive mission, UAVs evoke connotations in the minds of the public that borders on the absurd to highly valid concerns. Based on a recent study, it is expected that the market value will be a whopping USD 89 billion within the

T. Silva $\cdot$ F. Farina $\cdot$ R. Wiebbelling $\cdot$ L. Renner

Basin Modeling Laboratory, Institute of Geosciences, Federal University of Rio Grande do Sul, Porto Alegre, Brazil

e-mail: tatianasilva@ufrgs.br

F. Farina

e-mail: flavia.farina@ufrgs.br

R. Wiebbelling

e-mail: rfwiebbelling@gmail.com

L. Renner

e-mail: cardoso.renner@ufrgs.br

R. Madhavan $(\bowtie)$

Humanitarian Robotics and Automation Technologies, Clarksburg, USA

e-mail: rajmadhavan.t4h@gmail.com

E. Prestes

Phi Robotics, Institute of Informatics, Federal University of Rio Grande do Sul, Porto Alegre,

Brazil

e-mail: prestes@inf.ufrgs.br 
next 10 years (Drones: Market Overview 2014) Our take on UAVs is that they can be an invaluable tool and an asset in the sky providing images of unsurpassed quality in civilian applications ranging from disaster relief, environmental monitoring, and surveillance to combating animal poaching efforts and situational assessment and awareness.

Extreme events became obvious in Brazil in 2011 when the mountain region of Rio de Janeiro faced extreme rain in the order of $270 \mathrm{~mm}$ overnight. High slopes, clay soils, irregular settlements, and enormous amounts of rain lead to thousands of deaths and render dozens of thousands homeless (the official count is 35,000 but the actual number is much higher than that). This was the final outcome of the major hydrometeorological disaster in the Brazilian records. Rio Grande do Sul in Southern Brazil consists of several high-risk areas. Moreover, according to the Brazilian Civil Defense, almost 600 extreme events led municipalities to declare a state of emergency in Rio Grande do Sul between 2003 and 2010. Almost a million people were affected, either by becoming homeless or missing, getting injured, sick, dying, or having to relocate or in extreme cases, resulting in fatalities. It is not a matter of time before we are faced with floods and landslides. It is already happening. It is a matter of time until they happen regularly in such a scale that we start referring to them as disasters. Recent events lead us to the obvious conclusion: we were not prepared for that anywhere in Brazil. So the question is: how can we prepare, react, and adapt to this new environmental reality?

Among the diversity of natural environments around the world, certain ecosystems can be considered as vital areas, given their environmental functions in food production, hydrological control, sheltering, genetic stocking, etc. Hence, it is mandatory for our own survival to keep them protected to ensure the preservation of life support systems. However, this is not an easy task since significant manpower is needed to patrol thousands of hectares of land and water to control illegal fishing and hunting. In order to track global changes and their impact on the Earth system, we need specific tools and instruments, e.g., land use and cover mappings which are essential to model flood and landslide risks. A common way to perform these mappings is to use the information gathered by orbital remote sensing and/or cameras carried by manned aircraft. While the latter involves high costs and complicated logistics, the former cannot be used to support an emergency plan when a hydrological disaster occurs since its use imposes constraints like fixed revisit frequency; large elapsed time between an observation and the image supplied; and the presence of clouds.

An interesting and efficient solution is to use UAVs for obtaining a bird's-eye view of the landscape and also for capturing detailed and high-resolution images of the ground. UAVs can acquire high-resolution multispectral images, which in turn can produce 3D models of hazard zones without putting human lives at risk. Meteorologists, hydrologists, and spatial modelers can use such data to provide better detail and reliable results at a local scale. In this context, UAVs have the potential to assist in the acquisition of spatial data and also to evaluate the behavior, distribution, and size of the populations under study.

Our work with UAVs is centered on creating tools to support the preservation of human lives and the ecosystems that support them. In Taim Ecological Station 
located in Southern Brazil, Federal University of Rio Grande do Sul (UFRGS) and other stakeholders have acted collaboratively to assess geographic information to help the elaboration of an environmental plan to solve specific community demands and also to monitor the impact and dynamic aspects of the ecosystem, such as the occurrence of fire, invasive species, and environmental infractions.

We have developed Geographic Information Systems (GIS)-based regional models of environmental risk and how they overlap infrastructure and human settlements in the Rio Grande do Sul coastal zone (Silva et al. 2011; Silva and Tagliani 2012). Our current work focuses on enhancing the regional models using precision and super high-resolution images taken by UAVs especially for those areas already identified as high risk. The greater the precision of these models, the more lives can be saved because these models can form the basis for public policies in constraining human settlements on high-risk areas. Moreover, meteorological sensors carried by UAVs can be employed to collect atmospheric information, providing better precision for the meteorological models at a local level. When these data are integrated in hydraulic/hydrological models, scene-generation becomes possible, thus allowing us to predict which regions are vulnerable to floods or landslides depending on different levels of rainfall. This information can then aid rescue teams when a certain level of rainfall is expected once it is incorporated in collaborative platforms such as participatory WebGIS or similar ones.

This article details the development of an integrated framework that merges remote sensing capabilities (including but not limited to UAVs) using GIS models in a collaborative fashion with social capital. To the authors' knowledge, our efforts represent the first instance of the merging of multi-resolution remote sensing data, WebGIS, policy, and disaster management practices to arrive at an analytically rich, practical, and effective national disaster response structure for Brazil and possibly extendible to other developing economies. This article describes our work with UAVs for applications in environmental risk assessment, ecological monitoring, landslides/floods detection, and prediction, as well as regulatory aspects in Southern Brazil.

\subsection{Environmental Vulnerability and Susceptibility Studies}

Even if our eyes see the world in front of us as a colored 3D structure, it is much more than that. It is multidimensional. A single spot on the Earth's surface will have a certain level of vegetation density or it may be covered by urban settlement or buildings. And underneath it, you will find a certain type of soil, a water table at a certain depth, and, if you dig deeper, a certain type of rock. This spot will present an elevation value related to the sea level. And it may not be at the same plane related to as the sea level either but it can be present at a certain slope. Depending on the direction this slope-face (called "aspect"), this spot will receive a certain amount of sunlight and be more or less exposed to the wind forces. And all of these characteristics vary along the 3D space and through time. Besides that, the way water will flow when it rains depend on every one of these characteristics or dimensions. 
Part of it will infiltrate into the soil, part of it will run off, and part of it will evaporate. In a high slope spot covered by concrete, the water will run off. In a spot covered by a forest on a sandy soil at a low slope, water is going to infiltrate easily. So if a forest is replaced by agriculture, or even worse, by concrete, less water will infiltrate and, consequently, more water will run off. If water flows on the surface, it carries soluble substances, grains, small pieces of rock, big pieces of rock, cars, houses, and people. However, it is not enough to think in terms of a single spot, but we need to consider a region instead, and each one of these dimensions (vegetation, land use, soil, geology, slope, etc.) as superimposed layers.

GIS is the conventional platform to cross as many layers of information as needed so we can detect and model the spatial relationships between parameters. Once we formulate the (map) algebra, we can then generate digital maps depicting runoff and flow accumulation which accumulation, which, in turn, indicate landslide and flood risk. As noted earlier, spatial criteria change through time. We can use the same geology and soil maps as long as we live because they will not change for hundreds or thousands of years. But the same cannot be said about land use and cover. Forests turn into pasture. Natural fields turn into rice fields. Human activities are changing land cover ever faster. So fast that when a land use and cover map is done it is already outdated!

Remote sensing enables us to be quick in assessing this process. Sensors carried by satellites give us quantitative measures of the way a material interacts with the electromagnetic energy so we can differentiate targets based on what we call spectral response pattern. If a satellite carries a radar sensor, or yields stereo pairs, we can also obtain topographic data. This type of information comes as images, so we can mathematically manipulate them and find ways to automatically detect land use and cover changes. However, when we need very detailed spatial information, sometimes urgently, satellites present several constraints: low spatial resolution of free/low-cost images, high costs related to high spatial resolution images, a fixed revisit frequency, and requirements to schedule an observation, the time lapse between an observation and the image supply, or the simple presence of clouds.

What to do when we need to be very sure whether an extreme meteorological event will be hazardous or not for specific infrastructures or populations? Highresolution land use and cover maps, as well as 3D models, are required for hydrological, hydraulic, and meteorological models at a very local scale. In the imminence/occurrence of an extreme event, rapid assessment of high-risk/affected areas is needed. It would be way too cumbersome and labor-intensive to regularly map land cover and use and build 3D models with a high resolution (in the order of 2-5 m) for a region as large as $60,000 \mathrm{~km}^{2}$. This is the actual area of the Littoral Basin in Rio Grande do Sul where our efforts are focused (see Fig. 3.1). Besides, when it comes to map land use and cover, which is driven by human activities, we are pretty much always out of time. GIS models based on satellite data are great. They give us a broad view of vast regions so we can detect high-risk areas as well as where flood and landslide risks are increasing over time.

Our current work, thus, focuses on enhancing the regional models using precision and high-resolution images acquired using UAVs (Fig. 3.2), especially for those 

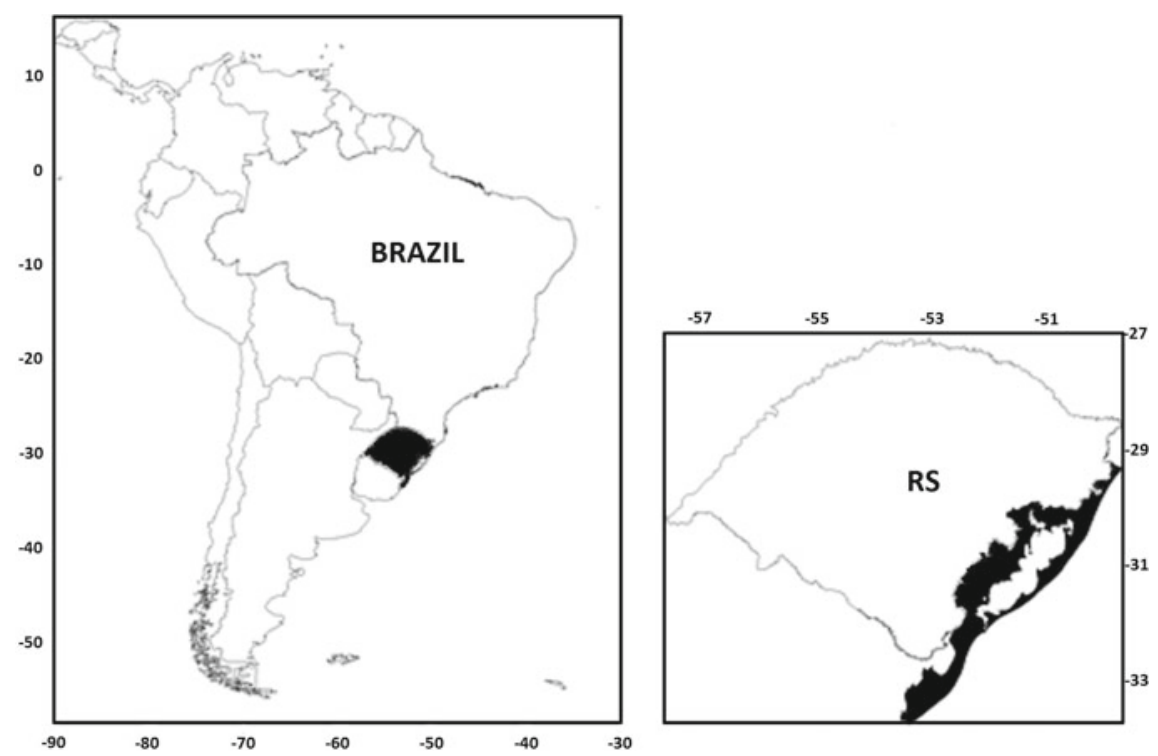

Fig. 3.1 Southern Brazil coastal plain

areas already identified as high risk. Robust, fixed wing UAVs are preferable in this phase as the goal is to acquire quality images as fast as possible. Satellite images with equivalent quality are costly and not so easy/fast to obtain for aforementioned reasons.

We deploy the ECHAR 20A UAVs developed by XMobots (XMobots), a Brazilian UAV manufacturer. It comprises very desirable characteristics: it flies up to $3 \mathrm{~km}$ altitude under wind speeds up to $45 \mathrm{~km} / \mathrm{h}$ and low rain, has $45 \mathrm{~min}$. autonomy, cruising speed of $75 \mathrm{~km} / \mathrm{h}$, stall speed of $37 \mathrm{~km} / \mathrm{h}$, visible RGB resolution $2.4-19.2 \mathrm{~cm}$. The challenge lies in the now relies in the image processing since ECHAR 20A yields 24 bits images with about 35,106 pixels per image. A 3D model based on images acquired using the UAVs provides the accuracy necessary to build detailed hydrological/hydraulic models.

The greater the precision of the input data, the greater the precision of the models, and consequently more lives can be saved because these models can be the basis for public policies in constraining human settlements on high-risk areas and to set off evacuation alerts. Moreover, meteorological sensors in the ECHAR 20A can be employed to collect atmospheric information thus providing better precision for the meteorological models at a local level. When these data are integrated into hydraulic/hydrological models, scenario generation becomes possible, as well as to predict which regions floods or landslides, depending on the levels of rainfall. This information also helps to anticipate the action of rescue teams when a certain level of rainfall is expected. 


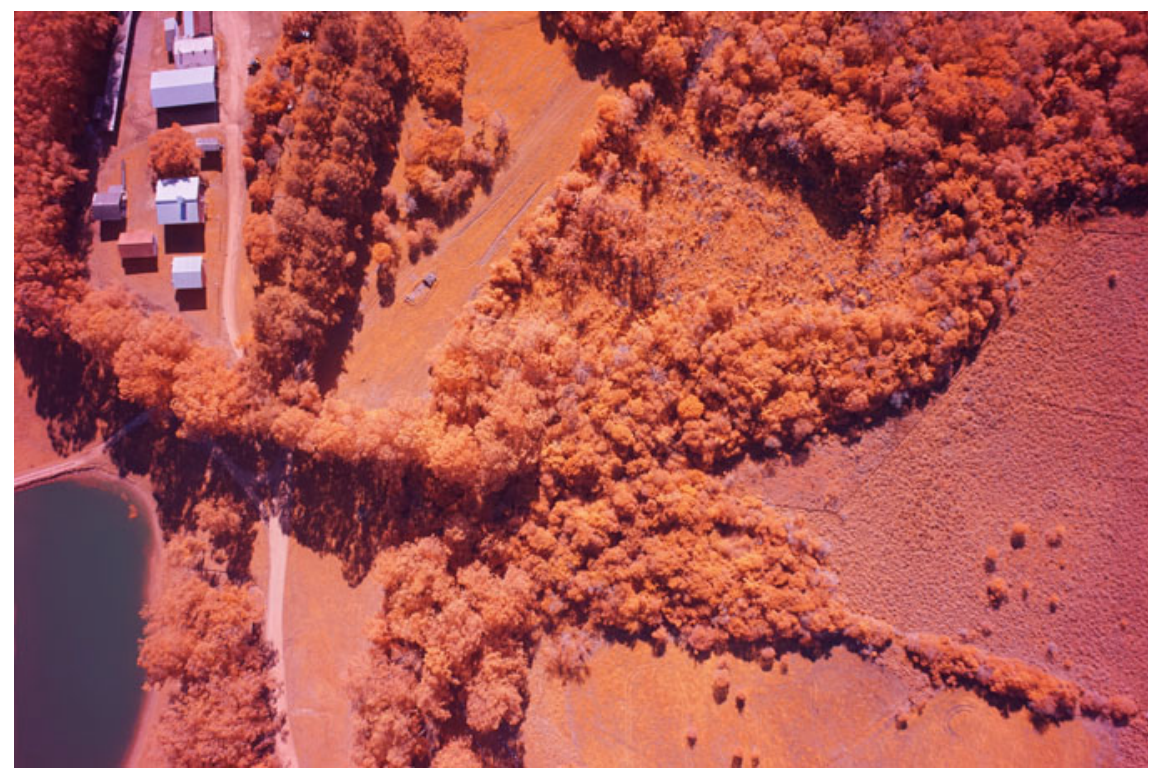

Fig. 3.2 A false color-composite image obtained by ECHAR 20A. Land use and cover mapping can be done with precision based on these images

The cataclysmic landslide in Rio de Janeiro provided some valuable lessons: Communication and field assessment are crucial. UAVs can replicate signals and enable communication when telecommunication towers collapse. UAVs can also be instrumental in planning alternative routes when roads and bridges are destroyed, and to (remotely) find people in need.

\subsection{Ecological Conservation: Mapping and Target Detection}

Land use and cover changes lead to soil impermeabilization. But we need land for agriculture, urban settlements, etc. Human disrupted ecosystems cannot replace these vital areas. They simply cannot play the same roles. Conservation units normally consist of vital areas. That means, ecosystems that play a vital role for the environmental functioning. Some of them are totally untouched; others have human uses more heavily regulated. It is not an easy task to monitor a conservation unit. It commonly means thousands of hectares of land and water to monitor and patrol. UAVs can provide the necessary information to better observe nature and to detect and prevent illegal activities, such as hunting and fishing.

Taim Ecological Station in Southern Brazil is an integral conservation unit. This unit spans a very wide wetland, internationally recognized by the RAMSAR 


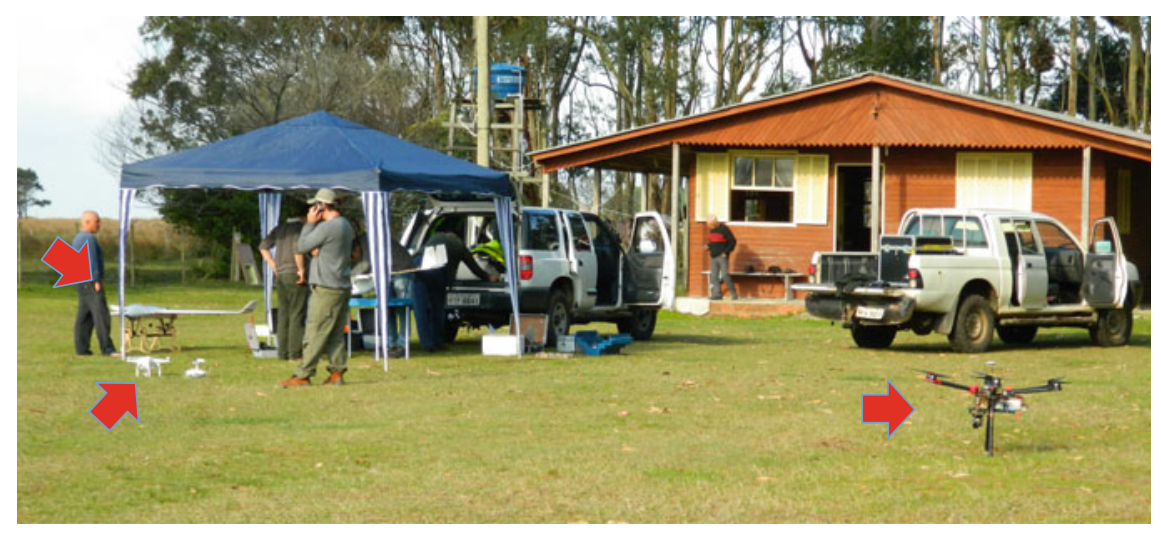

Fig. 3.3 Left to right: Lab-made fixed wing, Phantom 4, and DJI S-900

Convention (RAMSAR Convention). We have flown trial flights in this region but already have started to set mapping procedures to assess geographic information not available so far to support the decision making in two different situations:

(1) to help elaborating an environmental plan, which depends on mapping many spatial parameters with a high resolution, and

(2) to monitor impacts and dynamic aspects of the ecosystem. For the latter case, we have identified the need to build our own UAVs.

Figure 3.3 shows some of the UAVs often used in monitoring the Taim Station. Commercially available UAV platforms are usually not flexible enough for covering wide range of applications as those needed in managing and maintaining a conservation unit (e.g., land cover change detection, fauna and flora identification and monitoring, fire detection and monitoring, illegal fishing, and hunting detection). Besides, "lab-made" UAVs can reduce costs by more than ten times especially if a swarm of them need to be developed for distributed coverage of the vast land area. Since conservation units in Brazil can only rely on limited funding, it is imperative to bring forth affordable options of UAVs. Lab-made UAVs are being developed and is presented in last section. 


\subsection{Regulatory Constraints in Brazil}

Regulation is a crucial aspect of UAV usage in Brazil. UAVs weighting more than $150 \mathrm{~kg}$ are under the same regulations of airplanes. UAV between and $150 \mathrm{~kg}$ require a Brazilian Aeronautics Register and a formally habilitated pilot in performing any flight. UAVs with less than $25 \mathrm{~kg}$ need a register in the SISANT (Sistema de Aeronaves Não-Tripuladas/Unmanned Aerial Vehicles System), and also a habilitated pilot in performing flights above 400 feet. Flights above 400 feet also need to be authorized by ANAC (Agência Nacional de Aviação Civil/National Agency for Civil Aviation). Only UAVs under $250 \mathrm{~g}$ do not require anything to fly. Above that, UAVs must keep a minimum distance of $30 \mathrm{~m}$ from people, the pilot must be older than 18 years, and acquire a third-party damage insurance. In any situation, DECEA (Departamento de Controle do Espaço Aéreo/Aerial Space Control Department) rules must be followed. People close to the flight area must be aware and authorize the flight. Pilots of UAVs with $25 \mathrm{~kg}$ or more need a CMA (Certificado Médico Aeronáutico/Aeronautical Medical Certificate) emitted by ANAC or CMA. The use of UAVs for loads or goods transportation is forbidden. Only electronic devices such as cameras or computers are allowed during flight. The portage of the following documents is mandatory: flight manual, risk assessment document, and insurance. All these requirements and restrictions make it complicated to start applying UAVs in any field in Brazil, especially with bigger sensory payloads, which in general are more suitable for mapping purposes.

\subsection{Ongoing and Future Work}

GIS-modeling capabilities for UAV images, both for ecological conservation and disaster management, are currently being enhanced. For the Taim Ecological Station specifically, 3D models have been built, and some other applications are under study. The suitability of different UAVs for many management demands in Taim has been tested. These include capybara automatic counting (for population dynamics studies), fishing and hunting detection, nest detection, and bird identification. As mentioned before, low-cost UAV is the most desirable one for these cases, similar to the labmade ones that have been built (Fig. 3.3). The final goal in this sense is to define and create the most suitable "UAV family" to cover all types of technology gaps related to monitoring.

Social networks have emerged as an obvious way to exchange information during disasters. SMART (Simulation, Modeling, Analysis, Research, Teaching) Infrastructure Facility, part of the University of Wollongong in Australia, has used Twitter to trigger disaster alerts. Facebook now sends automatic messages enabling the user in a disaster area to post updates on his/her status. The next step in our work consists of integrating UAV data into such existing frameworks, as well as providing relevant spatial information accessible through WebGIS platforms. 


\section{References}

Drones: Market Overview. (2014). http://www.robolutioncapital.com.

RAMSAR Convention. The Convention on Wetlands. http://www.ramsar.org/.

Silva, T. S., De Freitas, D. M., Tagliani, P. R. A, Farina, F. C., \& Ayup-Zouain, R. N. (2011). Land use change impact on coastal vulnerability: subsidies for risk management and coastal adaptation. In Proceedings of International Symposium on GIS and Computer Mapping for Coastal Management (pp. 54-59).

Silva, T. S., \& Tagliani, P. R. A. (2012). Environmental planning in the medium littoral of the Rio Grande do Sul coastal plain Southern Brazil: Elements for coastal management. Ocean and Coastal Management, 59, 20-30.

XMobots. http://www.cpetecnologia.com.br/topografia/vant/547/echar-20a/xmobots.

Open Access This chapter is licensed under the terms of the Creative Commons Attribution 4.0 International License (http://creativecommons.org/licenses/by/4.0/), which permits use, sharing, adaptation, distribution and reproduction in any medium or format, as long as you give appropriate credit to the original author(s) and the source, provide a link to the Creative Commons license and indicate if changes were made.

The images or other third party material in this chapter are included in the chapter's Creative Commons license, unless indicated otherwise in a credit line to the material. If material is not included in the chapter's Creative Commons license and your intended use is not permitted by statutory regulation or exceeds the permitted use, you will need to obtain permission directly from the copyright holder.

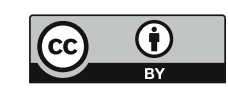

